\title{
The value of plasma SCUBE1 and oxidative stress parameters in the early diagnosis of acute ovarian torsion
}

\author{
Gunaydin $\mathrm{M}^{1}$, Sipahi $\mathrm{M}^{2}$, Kesicioglu $\mathrm{T}^{3}$, Usta $\mathrm{M}^{4}$, Tezcan $\mathrm{B}^{5}$, Tokgoz VY ${ }^{6}$ \\ Giresun University, Faculty of Medicine, Department of Emergency Medicine, Giresun, Turkey. \\ mgunaydin@hotmail.com
}

\begin{abstract}
AIM: The purpose of our study was to determine the diagnostic value of plasma SCUBE1, malondialdehyde (MDA), superoxide dismutase (SOD) and total antioxidant status (TAS) investigated on the $4^{\text {th }}$ hour of ovarian torsion, and at the same time to determine the relation between these markers and histopathological damage occurring in the ovary.

MATERIALS AND METHODS: Sixteen rats were included, eight in the torsion group and eight in the control group. The right ovaries of the rats in the torsion group were rotated clockwise 720 degrees, after which they were fixed and attached to the abdominal wall. The abdominal walls of the control group rats were opened for $1 \mathrm{~min}$ and then closed. Relaparotomy was performed $4 \mathrm{~h}$ after surgery in both groups, blood specimens were collected for biochemical analyses, and the right ovaries were surgically extracted.

RESULTS: No statistically significant difference was determined in the control and torsion groups' plasma SCUBE1, SOD and TAS values $(p=0.987, p=0.33$, and $p=0.244$, respectively). Torsion group plasma MDA values were significantly higher than those of the control group $(p=0.039)$. At histopathological evaluation, histological score, apoptotic index and Caspase-3 index values of the torsion group were significantly higher compared to the control group.

CONCLUSION: Plasma SCUBE1 is not useful in the early diagnosis of ovarian torsion. Similarly, use of the oxidative stress markers SOD and TAS does not appear useful in early diagnosis, although MDA may be considered for that purpose (Tab. 2, Fig. 3, Ref. 23). Text in PDF www.elis.sk.

KEY WORDS: ovarian torsion, SCUBE1, oxidative stress.
\end{abstract}

\section{Introduction}

Ovarian torsion is a gynecological emergency that can be seen in women of all ages. However, it is most common in the reproductive age group (1). It is caused by complete or partial rotation of organs supporting the adnexa, which results in ischemic changes in the ovary (2). Since definite diagnosis is only possible during surgery, the true incidence is unknown. However, the annual prevalence is between $2 \%$ and $6 \%$ (3). Ovarian torsion is estimated in approximately $3 \%$ of patients presenting to the emergency

${ }^{1}$ Giresun University Faculty of Medicine, Department of Emergency Medicine, Giresun, Turkey, ${ }^{2}$ Giresun University Faculty of Medicine, Department of Obstetrics and Gynecology, Giresun, Turkey, ${ }^{3}$ Giresun University Faculty of Medicine, Department of General Surgery, Giresun, Turkey, ${ }^{4}$ Giresun University Faculty of Medicine, Department of Medical Biochemistry, Giresun, Turkey, ${ }^{5}$ Giresun University Faculty of Medicine, Department of Histology and Embryology, Giresun, Turkey, and ${ }^{6}$ Eskisehir Osmangazi University Faculty of Medicine, Department of Obstetrics and Gynecology, Eskisehir, Turkey

Address for correspondence: M. Gunaydin, MD, Giresun University, Faculty of Medicine, Department of Emergency Medicine, Giresun Universitesi, Tip Fakultesi Dekanligi, Gazipasa Yerleskesi, Debboy Mevki, 28100, Giresun, Turkiye.

Phone: +90.454 .3101600 , Fax: +90.454 .3101690$

Acknowledgement: The study was supported by the Scientific Research Committee of Giresun University (100517-125). department with acute abdominal pain (4). Delays in diagnosis and treatment can result in ovarian losses, and early diagnosis is therefore important in terms of preventing complications (5). No specific laboratory marker for routine use in clinical practice in the preoperative diagnosis of ovarian torsion has to date been identified. Studies involving interleukin-6 (IL-6), ischemia-modified albumin (IMA), D-Dimer and pentraxin-3 have determined that these markers may be related to ovarian torsion (6-10).

Signal peptide-CUB (complement $\mathrm{C} 1 \mathrm{r} / \mathrm{C} 1 \mathrm{~s}$, Uegf, and Bmp1)-EGF (epidermal growth factor)-like domain-containing protein-1 (SCUBE1) is a cell surface protein released throughout early embryogenesis. It is released by the endothelium and platelets and is a potential marker showing platelet activation (11). These molecules are deposited inside alpha granules in inactivated platelets, are translocated to the platelet surface following activation by thrombin, are released in the form of small soluble particles, and are incorporated into thrombus (12). Previous studies have determined that SCUBE1 increases in conditions resulting in platelet activation and ischemic complications, such as acute pulmonary embolism, acute ischemic stroke, and acute testicular torsion (13-15)

Oxidative stress is defined as impairment of oxidative homeostasis as a result of an increase in reactive oxygen species (ROS) forming during cell metabolism and of insufficient levels of antioxidants that detoxify these. The ROS forming as a result 
of increased oxidative stress initiate lipid peroxidation by causing oxidation in polyunsaturated acids in biological membranes. Biochemical measurement of malondialdehyde (MDA), the end product of lipid peroxidation, in body fluids and tissues is used to identify the presence of oxidative damage (16). Studies have determined that MDA levels rise significantly in line with the duration of the ischemic process (17). Superoxide dismutase (SOD) is an endogenous antioxidant enzyme and one of the defense mechanisms known as antioxidants that serve to prevent the damage to cellular structures caused by ROS. SOD assists with the prevention of ischemia- and perfusion-related tissue damage, and exhibits a beneficial effect under these conditions (18). Total antioxidant status (TAS) reflects the organism's total antioxidant defense against free radical attack in plasma. TAS measurement is a useful test for estimating oxidative status (17).

The purpose of this study was to determine the diagnostic value in ovarian torsion of plasma SCUBE1 and of the oxidative stress markers MDA, SOD and TAS, and also to investigate the relation between these markers and histopathological damage occurring simultaneously in the ovary.

\section{Materials and methods}

\section{Animals and study design:}

Sixteen adult female Wister albino rats weighing 250-300 g were used in this experimental study. Before the study began, all animals were kept in separate cages for one week, under fixed temperature and humidity, in a 12-h light-dark cycle, with ad libitum access to food. The study commenced following approval from the Animal Experiments Local Ethical Committee. The rats were randomized on the day of the experiment into two groups, torsion and control, each consisting of eight animals.

\section{Anesthesia and surgical technique}

After being weighed, intraperitoneal ketamine hydrochloride (50 mg/kg Ketalar, Eczacibaşi, Istanbul, Turkey) and xylazine hydrochloride $(10 \mathrm{mg} / \mathrm{kg}$, Rompun, Bayer Türk Ilaç Ltd, Istanbul, Turkey) were administered for anesthesia, repeated as required for maintenance of anesthesia during the experiment. Rats were not intubated during the study, but were allowed to breathe spontaneously.

For surgery, rats were placed in a supine position and covered with a sterile surgical cloth. The incision site was then shaved and disinfected. A 2-cm longitudinal incision was made in the abdominal midline for laparotomy, and the uterine horns and adnexa were exposed. In the control group, the abdominal wall was opened for $1 \mathrm{~min}$ and then closed with 3/0 silk suture. In the torsion group, the right ovary was rotated 720 degrees and fixed to the abdominal wall with $4 / 0$ silk suture. The incision was then closed with $3 / 0$ silk suture.

Relaparotomy was performed in both groups from the previous incision line 4 hours after the first operation. Approximately 1.5-2 mm blood specimens were also collected from the abdominal aorta for biochemical analyses. Following collection of blood specimens, the right ovaries of rats in both groups were surgically extracted. All rats were finally sacrificed by cervical dislocation once the surgical procedures had been completed.

\section{Biochemical analysis}

Blood specimens were placed into tubes containing heparin as a coagulant, after which they were centrifuged for $20 \mathrm{~min}$ at $1000 \mathrm{xg}$ and $4{ }^{\circ} \mathrm{C}$. The supernatants obtained were aliquoted and stored at $-80^{\circ} \mathrm{C}$ until the day of analysis.

For SOD activity analysis, plasmas were kept on ice and studied using a commercial kit (Superoxide Dismutase Assay Kit, Cayman Chemical Co., Ann Arbor, MI, USA, Batch: 0525723), as described by the manufacturer. For lipid peroxidation evaluation, plasma MDA levels were measured based on the principle of thiobarbituric acid reactive substances (TBARS) formation at high temperature and in an acidic environment using a commercial kit (TBARS Assay Kit, Cayman Chemical, Ann Arbor, MI, USA, Batch: 0525498) as recommended. Plasma MDA levels were expressed as mmol/L. Total antioxidant status (TAS) was assessed based on reduction of the dark blue-green 2,2'-azino-bis(3ethylbenzothiazoline-6-sufhonic acid) radical cation $\left(\right.$ ABTS $^{*+}$ ) of the antioxidants in the plasma specimen to the colorless reduced ABTS form (16). ( \pm )-6-Hydroxy-2,5,7,8-tetramethylchromane2-carboxylic acid (Trolox Equivalent), a Vitamin E analogue, was used as standard. Results were expressed as mmol Trolox Equiv./L.

Plasma SCUBE1 levels were measured using an enzymelinked immunosorbent assay reagent kit (Cloud-Clone Corp., 23603 W. Fernhurst Dr., Unit 2201, Katy, TX 77494, USA, Lot: L180307290), and were expressed as $\mathrm{ng} / \mathrm{mL}$.

\section{Histological analysis}

The extracted right ovaries were rapidly fixed by being placed in $10 \%$ neutral formalin solution. Following histological tissue fixation and embedding in paraffin, $5-\mu \mathrm{m}$ thick sections were taken from the blocks. Sections were fist stained with hematoxylin eosin (HE) dye in order to examine tissues' histological structures. Tissues were subjected to histological analysis under light microscopy and scored between 1 and 3 based on parameters of follicular cell degeneration, vascular congestion, inflammatory cell infiltration and hemorrhagic damage: 0 ; no pathological finding in the ovarian section. 1: Mild; pathological findings in less than $33 \%$ of the ovarian section, 2: Moderate; pathological findings in 33-66\% of the ovarian section, and 3: Severe, pathological findings in more than $66 \%$ of the ovarian section.

TUNEL (Merck) and Caspase 3 (Santa Cruz Biotechnology, Inc.) immunofluorescent staining were performed in line with the manufacturers' protocols in order to determine differences in apoptosis between the control and torsion groups. Immunopositive stained and unstained cells in each section were counted through random selection of five areas under fluorescent microscope at 40X magnification. The proportion of Immunopositive-stained cells to the total number of cells in an area (apoptotic index and Caspase-3 index) was calculated using the following formulae: Apoptotic index $=$ [apoptotic cell count/total cell count $] \times 100$, and Caspase- 3 index = [Caspase- 3 immunopositive cell count $/$ total cell count] 100 . 
Tab. 1. Biochemistry results and ovarian tissue histological scores and apoptotic index and Caspase- 3 index results from the control and torsion groups.

\begin{tabular}{lccc}
\hline Plasma & $\begin{array}{c}\text { Control group } \\
(\mathrm{n}=8)\end{array}$ & $\begin{array}{c}\text { Torsion group } \\
(\mathrm{n}=8)\end{array}$ & $\mathrm{p}$ \\
\hline SCUBE1 $(\mathrm{ng} / \mathrm{mL})$ & $1.82 \pm 0.16$ & $1.82 \pm 0.18$ & 0.987 \\
SOD $(\mathrm{U} / \mathrm{mL})$ & $5.33 \pm 0.44$ & $5.98 \pm 0.45$ & 0.33 \\
MDA (mmol/L) & $25.81 \pm 2.16$ & $33.83 \pm 2.78$ & 0.039 \\
TAS (mmol Trolox Equiv./L) & $0.92 \pm 0.04$ & $1.04 \pm 0.08$ & 0.244 \\
\hline Ovarian tissue & & & \\
\hline Histological score & $0.38 \pm 0.183$ & $2.63 \pm 0.183$ & $<0.001$ \\
Apoptotic index & $1.209 \pm 0.159$ & $4.807 \pm 0.361$ & $<0.001$ \\
Caspase-3 index & $2.127 \pm 0.087$ & $7.689 \pm 0.416$ & $<0.001$ \\
\hline
\end{tabular}

All values are presented as mean \pm SD. SCUBE1 $=$ Signal peptide-CUB (complement $\mathrm{C} 1 \mathrm{r} / \mathrm{C} 1 \mathrm{~s}$, Uegf, and Bmp1)-EGF (epidermal growth factor)-like domain-containing protein- $1, \mathrm{SOD}=$ superoxide dismutase, $\mathrm{MDA}=$ malondialdehyde, $\mathrm{TAS}=$ total antioxidant status.

\section{Statistical analysis}

SPSS software was used for statistical analyses. Compatibility of normal distribution of data was assessed using the Shapiro-Wilk test. Normally distributed data were expresses as mean/standard deviation. Student's t test was used for comparisons between the torsion and control groups. Relations between biochemical data and histopathological findings were investigated using Pearson correlation analysis. Results were evaluated at a $95 \%$ confidence interval and at a significance level of $\mathrm{p}<0.05$.

\section{Results}

\section{Biochemical results}

Results for plasma SCUBE1, SOD, MDA and TAS investigated from blood specimens collected from the torsion and control groups are shown in Table 1. No significant difference was determined in plasma SCUBE1, SOD and TAS between the two groups $(\mathrm{p}=0.987, \mathrm{p}=0.33$, and $\mathrm{p}=0.244$, respectively). Plasma MDA values were significantly higher in the torsion group than in the control group $(\mathrm{p}=0.039)$.

\section{Histopathological findings}

Sections taken from paraffin blocks from the torsion and control groups were analyzed under light microscope following staining with HE and then compared. Degeneration in follicular cells, vascular congestion, inflammatory cell infiltration and hemorrhage were observed in the torsion group compared to the control group (Fig. 1). Separate histological scoring of the sections produced a significantly higher value in the torsion group than in the control group ( $\mathrm{p} \leq 0.001)$ (Tab. 1).
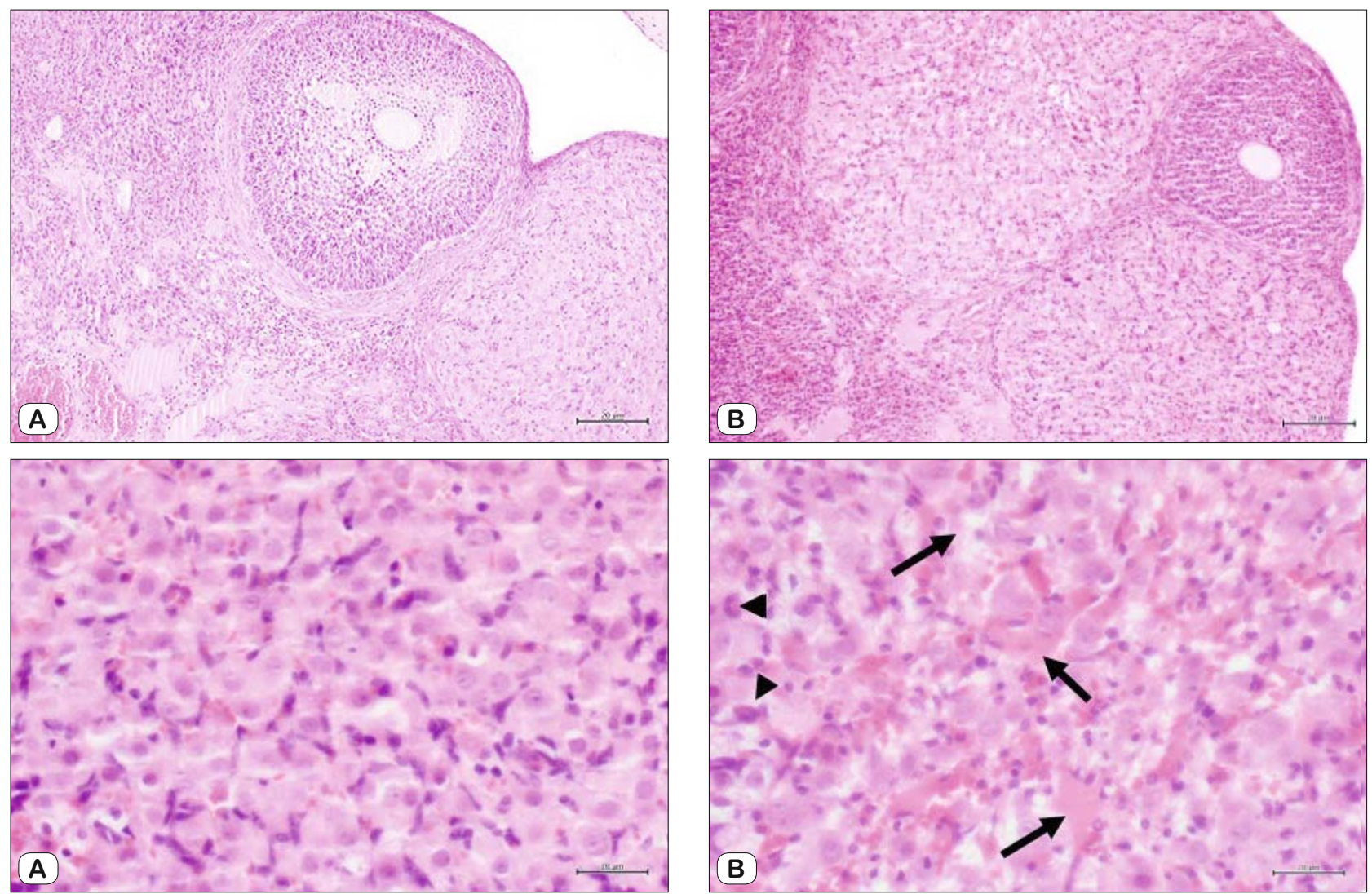

Fig. 1. Light microscopy images of control (A) and torsion (B) group hematoxylin eosin stained ovarian tissues at $10 \mathrm{X}$ and $40 \mathrm{X}$ magnification. Arrows indicate vascular congestion and hemorrhagic areas, while arrow heads indicate inflammatory cell infiltration. 

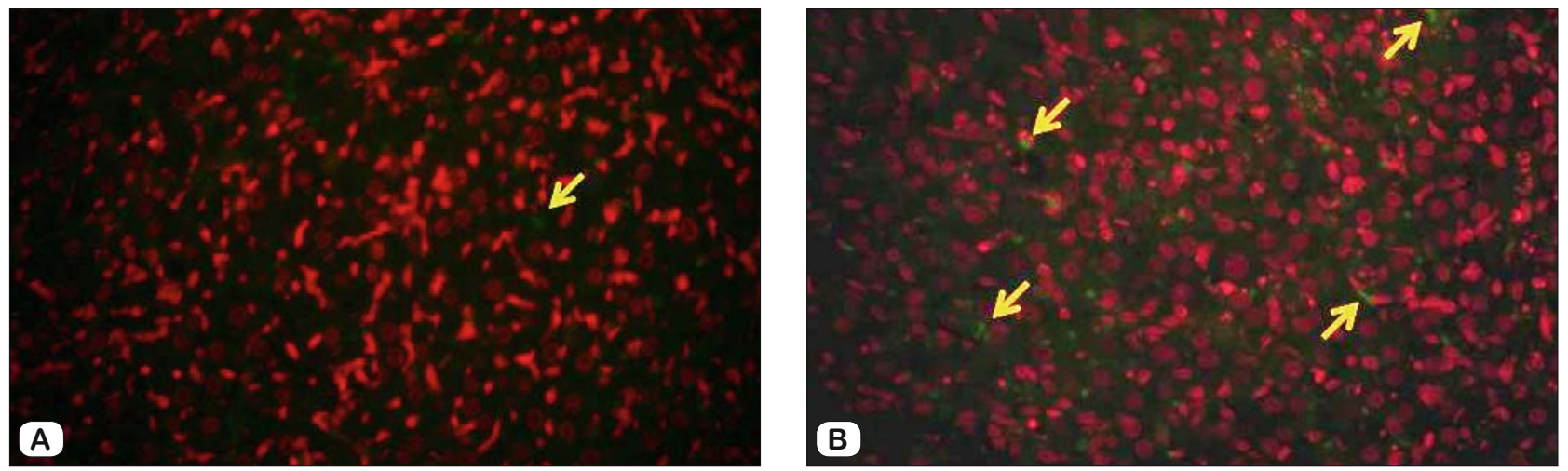

Fig. 2. Immunofluorescence staining using the TUNEL method in ovarian tissues from the control (A) and torsion (B) groups under fluorescent microscope at 40X magnification. Arrows indicate immunopositive-stained cells.
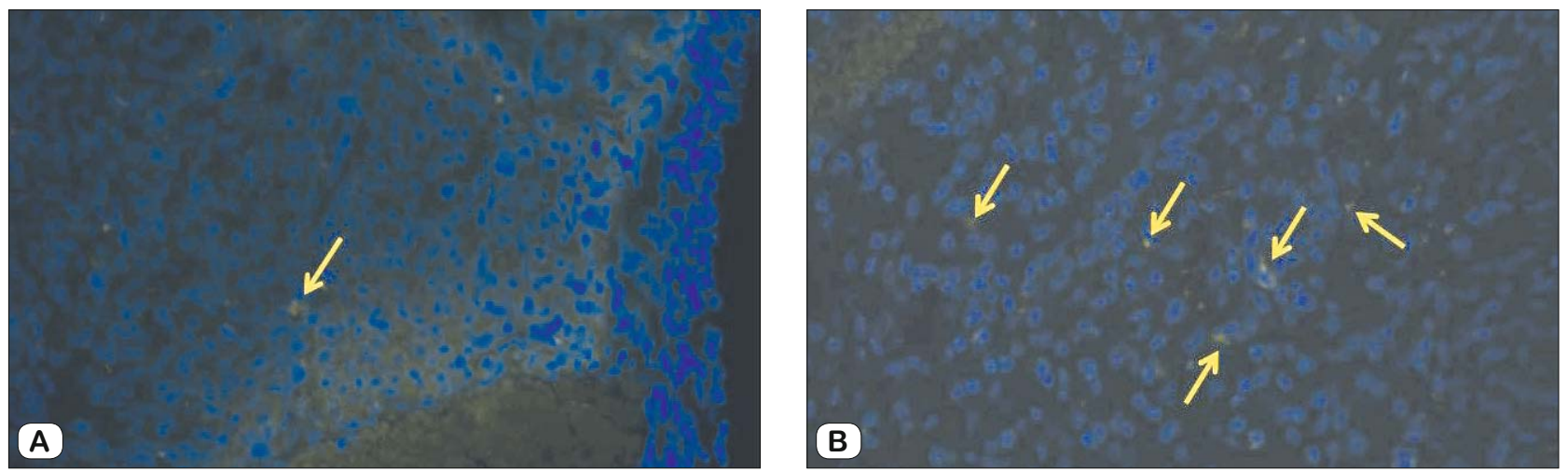

Fig. 3. Caspase-3 immunofluorescent staining of ovarian tissues from the control (A) and torsion (B) groups under fluorescent microscope at 40X magnification. Arrows indicate immunopositive-stained cells.

\section{Immunohistochemical findings}

Sections subjected to immunofluorescent staining with the TUNEL method and sections subjected to Caspase-3 immunofluorescent staining were examined under a light microscope and photographed (Figs 2, 3). Apoptotic index and Caspase-3 index values were calculated by performing cell counts in five random areas on each section. Apoptotic index and Caspase- 3 index values were significantly higher in the torsion group than in the control group ( $\mathrm{p} \leq 0.001$ for both) (Tab. 1).

In addition, correlation analysis revealed no statistically significant relations between plasma SCUBE1, SOD, MDA and TAS values and histological scores or apoptotic index and Caspase-3 index values (Tab. 2).

\section{Discussion}

In this experimental study, we determined plasma SCUBE1, MDA, SOD and TAS values 4 hours after induction of ovarian torsion, together with histological score, apoptotic index and Caspase-3 index values, and compared these with control group data. We observed no statistically significant differences between torsion group plasma SCUBE1, SOD and TAS values compared with the control group, and only MDA values were significantly higher in the control group. Histological score, apoptotic index and Caspase-3 index values were significantly higher than in the control group. In addition, we determined no statistically significant correlations between plasma SCUBE1, SOD, MDA and TAS values and histological scores or apoptotic index and Caspase-3 index values.

Tab. 2. Correlations between plasma SCUBE1, SOD, MDA and TAS levels and histological score and apoptotic index and Caspase- 3 index values in the torsion group.

\begin{tabular}{llccc}
\hline & & $\begin{array}{c}\text { Histological } \\
\text { score }\end{array}$ & $\begin{array}{c}\text { Apoptotic } \\
\text { index }\end{array}$ & $\begin{array}{c}\text { Caspase-3 } \\
\text { index }\end{array}$ \\
\hline SCUBE1 & r value & -0.213 & -0.162 & -0.585 \\
$(\mathrm{ng} / \mathrm{mL})$ & p value & 0.612 & 0.702 & 0.127 \\
\hline $\mathrm{SOD}$ & $\mathrm{r}$ value & 0.149 & -0.149 & -0.207 \\
$(\mathrm{U} / \mathrm{mL})$ & $\mathrm{p}$ value & 0.725 & 0.724 & 0.623 \\
\hline $\mathrm{MDA}$ & r value & 0.116 & -0.234 & 0.062 \\
$(\mathrm{mmol} / \mathrm{L})$ & $\mathrm{p}$ value & 0.784 & 0.577 & 0.883 \\
\hline TAS $(\mathrm{mmol}$ & $\mathrm{r}$ value & 0.206 & 0.069 & 0.413 \\
Trolox Equiv/L) & $\mathrm{p}$ value & 0.624 & 0.872 & 0.309 \\
\hline
\end{tabular}

SCUBE1 = Signal peptide-CUB (complement C1r/C1s, Uegf, and Bmp1)-EGF (epidermal growth factor)-like domain-containing protein-1, SOD = superoxide dismutase, $\mathrm{MDA}=$ malondialdehyde, $\mathrm{TAS}=$ total antioxidant status. 
456-461

Ischemic changes occur in the ovary during ovarian torsion. Venous flow and lymphatic return are first blocked. Diffuse expansion in the ovarian parenchyma, edema and peripheral follicular extension are observed with continuing arterial perfusion. Venous obstruction followed by arterial thrombosis subsequently occur due to increasing edema and pressure $(8,9)$. Since ovarian torsion is frequently seen in young women, the ovary may be lost due to delays in diagnosis and surgical treatment, and fertility problems may be observed in these patients. Early diagnosis is therefore very important in terms of preventing complications (1, 4). Despite the use of imaging techniques such as ultrasonography, Doppler ultrasonography, and computerized tomography, diagnosis is still difficult because of non-specific findings in ovarian torsion (19). Although studies have investigated various markers of potential use in the early preoperative diagnosis of ovarian torsion, no specific marker has been identified. SCUBE1 is a reliable marker of platelet activation. Previous studies have shown that SCUBE1 levels increase under conditions of platelet activation and aggregation resulting in ischemic complications $(12,13,15)$. Turkmen et al. determined that SCUBE1 levels increase rapidly in the $2 \mathrm{nd} \mathrm{h}$ following acute mesenteric ischemia, rising significantly by $6 \mathrm{~h}$, while Turedi et al. showed that SCUBE1 increase significantly $4 \mathrm{~h}$ after testicular torsion $(15,17)$. In the present study, we determined no significant increase in plasma SCUBE1 levels $4 \mathrm{~h}$ after ovarian torsion compared to the control group, despite the mechanism being similar to that in testicular torsion. From that perspective, our study is not compatible with those of Turedi and Turkmen. Two recent studies, one experimental and the other clinical, have investigated the relation between ovarian torsion and plasma SCUBE1. Uzun et al. determined no significant increase in 8th-h plasma SCUBE1 levels in rats with induced ovarian torsion compared to a control group, although SCUBE1 levels increased significantly at $24 \mathrm{~h}$ (20). In their clinical study of patients diagnosed with ovarian torsion and undergoing laparotomy, Uyanikoglu et al determined mean torsion times of 24.4 $\pm 14.8 \mathrm{~h}$ and observed significantly higher preoperative SCUBE1 levels in these patients compared to healthy women (21). Considering our findings together with those of Uzun and Uyanikoglu, it may be concluded that plasma SCUBE1 levels do not increase in the early hours of ovarian torsion, but rise significantly at 24 h. Studies have revealed venous and lymphatic congestion in the early period of ovarian torsion, although total arterial occlusion may not be present, for which reason ovarian tissue may remain viable even at $24 \mathrm{~h}$, while necrosis begins at $36 \mathrm{~h}(7,22)$. The absence of an increase in SCUBE1 $4 \mathrm{~h}$ following ovarian torsion in the present study may be due to arterial circulation in ovarian tissue not being compromised in the early hours of torsion.

We also investigated parameters indicating oxidative status. Precious studies have revealed that oxidative stress is activated during ovarian torsion (23). We observed no significant change in plasma SOD and TAS investigated at $4 \mathrm{~h}$ during ovarian torsion in our study, with only MDA levels increasing significantly. Histopathological analysis revealed degeneration in follicular cells, vascular congestion, inflammatory cell infiltration and hemorrhage in the torsion group, while torsion group histological scores and apoptotic index and Caspase-3 index values were significantly higher than those of the control group. These findings show that our model of ovarian torsion was established successfully. However, we determined no significant correlation between biochemical and histopathological data in our study. The fact that biochemical data studied from serum were not compatible with the histopathological data may probably be attributed to ischemia not developing fully due to absence of complete arterial occlusion because of the mechanism involved in ovarian torsion in the early hours, and to findings not being reflected in blood at $4 \mathrm{~h}$.

\section{Limitation}

The principal limitation of this study is that preoperative biochemical data could not be analyzed since preoperative blood was not collected from the torsion and control groups. Another limitation is that biochemical data were not investigated using tissue homogenization.

\section{Conclusion}

Our study findings indicate no significant increase in plasma SCUBE1 levels investigated at 4 hours in ovarian torsion. We therefore think that plasma SCUBE1 levels are not useful in the early diagnosis of ovarian torsion. Similarly, we also think that the oxidative stress markers SOD and TAS are not useful in the early diagnosis of ovarian torsion, but that MDA may be capable of use for this purpose.

\section{References}

1. Huchon C, Fauconnier A. Adnexal torsion: A literature review. Eur J Obstet Gynecol Reprod Biol 2010; 150: 8-12.

2. Ding DC, Hsu S, Kao SP. Isolated torsion of the hydrosalpinx in a postmenopausal woman. JSLS 2007; 11: 252-254.

3. Anteby S, Schenker J, Polishuk W. The value of laparoscopy in acute pelvic pain. Ann Surg 1975; 181: 484-486.

4. Sasaki KJ, Miller CE. Adnexal torsion: review of the literature. J Minim Invasive Gynecol 2014 Mar-Apr; 21 (2): 196-202

5. Bayer AI, Wiskind AK. Adnexal torsion: can the adnexa be saved. Am J Obstet Gynecol 1994; 171: 1506-1511.

6. Cohen SB, Wattiez A, Stockheim D, Seidman DS, Lidor AL, Mashiach S, Goldenberg M. The accuracy of serum interleukin-6 and tumour necrosis factor as markers for ovarian torsion. Hum Reprod 2001; 16: 2195-2197.

7. Daponte A, Pournaras S, Hadjichristodoulou C, Lialios G, Kallitsaris A, Maniatis AN, Messinis IE. Novel serum inflammatory markers in patients with adnexal mass who had surgery for ovarian torsion. Fertil Steril 2006; 85: 1469-1472.

8. Aran T, Guven S, Unsal MA, Alver A, Mentese A, Yulug E. Serum ischemia-modified albumin as a novel marker of ovarian torsion: an experimental study. Eur J Obstet Gynecol Reprod Biol 2010; 150: 72-75.

9. Kart C, Aran T, Guven S, Karahan SC, Yulug E. Acute increase in plasma D-dimer level in ovarian torsion: an experimental study. Hum Reprod 2011 Mar; 26 (3): 564-568. 
10. Akman L, Erbas O, Terek MC, Aktug H, Taskiran D, Askar N. The long pentraxin-3 is a useful marker for diagnosis of ovarian torsion: An experimental rat model. J Obstet Gynaecol 2016; 36 (3): 399-402.

11. Tu CF, Yan YT, Wu SY, Djoko B, Tsai MT, Cheng CJ, Yang RB. Domain and functional analysis of a novel platelet-endothelial cell surface protein, SCUBE1. J Biol Chem 2008; 283: 12478-12488.

12. Dai DF, Thajeb P,Tu CF, Chiang FT, Chen CH, Yang RB, Chen JJ. J Am Coll Cardiol 2008; 51: 2173-2180.

13. Turkmen S, Sahin A, Gunaydin M, Sahin S, Mentese A, Turedi S, Karahan SC, Ozsu S, Gunduz A. The value of signal peptide-CUB-EGF domain-containing protein-1 (SCUBE1) in the diagnosis of pulmonary embolism: a preliminary study. Acad Emerg Med 2015; 22 (8): 922-926.

14. Turkmen S, Eryigit U, Karaca Y, Mentese A, Sumer UA, Yulug E, Aksut N, Gazioglu S, Gunduz A. Diagnostic value of plasma signal peptide-Cub-Egf domain-containing protein-1 (SCUBE-1) in an experimental model of acute ischemic stroke. Am J Emerg Med 2015; 33 (2): 262-265.

15. Turedi S, Tatli O, Alver A, Karaguzel E, Karaca Y, Turkmen S, Yulug E, Sumer A, Altay DU, Mentese A. The Diagnostic Value of Plasma SCUBE1, a Novel Biomarker of Platelet Activation in Testicular Torsion: A Randomized, Controlled, Experimental Study. Urology 2015; 86 (3): 516-520.

16. Ozcan O, Erdal H, Cakirca G, Yonden Z. Oxidative stres and its impact on intracellular lipids, protein and DNA. J Clin İnvest 2015; 6 (3): 331-336.
17. Turkmen S, Mentese S, Mentese A, Sumer AU, Saglam K, Yulug E, Turedi S, Gunduz A. The value of signal peptide-CUB-EGF domaincontaining protein 1 and oxidative stress parameters in the diagnosis of acute mesenteric ischemia. Acad Emerg Med 2013; 20: 257-264.

18. Salvemini D, Cuzzocrea S. Superoxide, superoxide dismutase and ischemic injury. Curr Opin Investig Drugs 2002; 3: 886-895.

19. Wilkinson C, Sanderson A. Adnexal torsion: a multimodality imaging review. Clin Radiol 2012; 67: 476-483.

20. Uzun Ö, Kaban I, Midi A, Uysal H, Boran AB, Bacanakgil BH, Tarbaghia M. Diagnostic value of signal peptide-CUB-EGF domaincontaining protein 1 as an early and late biochemical marker in the ovarian torsion rat model. J Obstet Gynaecol Res 2018; 44 (6): 1092-1099.

21. Uyanikoglu H, Hilali NG, Yardimciel M, Koyuncu I. A new biomarker for the early diagnosis of ovarian torsion: SCUBE-1. Clin Exp Reprod Med 2018; 45 (2): 94-99.

22. Taskin O, Birincioglu M, Aydin A, Buhur A, Burak F, Yilmaz I, Wheeler JM. The effects of twisted ischaemic adnexa managed by detorsion on ovarian viability and histology: An ischaemia-reperfusion rodent model. Hum Reprod 1998; 13: 2823-2827.

23. Kilic A, Uyanikoglu H, Incebiyik A. Protective effect of N-acetylcystein and resveratrol on ischemia-reperfusion injury in rat ovary. Dicle Med J 2016; 43: 229-236.

Received March 3, 2019. Accepted April 5, 2019. 